\title{
Balancing the pruning programmes
}

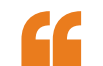

DUSP 16 acts

as a negative regulator of the apoptotic system through PUMA and acts as a positive regulator of axon morphogenesis and sensory axon development

During development, dorsal root ganglion (DRG) neurons compete for limited amounts of target-derived trophic factors such as nerve growth factor (NGF), which regulates neuronal survival and target innervation. Loss of trophic signalling results in axonal pruning by degeneration. This degeneration process is executed by transcriptional regulation of apoptotic pathway, but the precise nature of this transcriptional programme has not been elucidated. Now, Maor-Nof et al. show that dual specificity protein phosphatase 16 (DUSP16) plays a crucial part in regulating the balance between axonal preservation and axonal degeneration during development.

The authors used a model in which sensory DRG neurons from mice at embryonic day 13.5 (E13.5) were cultured for 36 hours with NGF, after which NGF was removed to induce

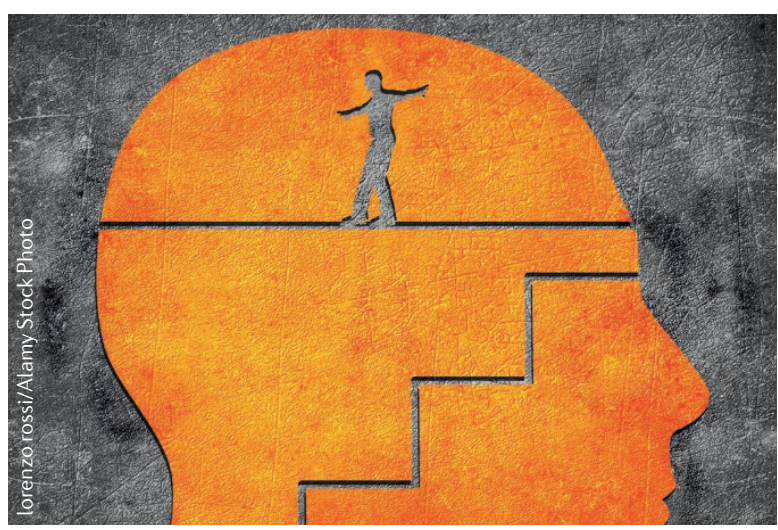

trophic withdrawal and axonal degeneration. This protocol upregulated expression of cell death-related genes (including Dusp16 and p53 upregulated modulator of apoptosis (Puma)) and downregulated the expression of cell survival-related genes, with these changes peaking at 3-6h after NGF withdrawal. Interestingly, a few components of this transcriptional programme were shared with nerve injury-induced genes, one of them being Dusp16. As little is known about the function of DUSP16 in neural development, the authors generated Dusp $16^{-/-}$mice and found that axonal degeneration in embryonic DRG neurons from these mice induced by trophic withdrawal occurred faster than in controls. Mutant DRG neurons subjected to trophic withdrawal also showed higher levels of the activated form of the apoptotic mediator caspase 3 , higher levels of phosphorylated (activated) p53 and more rapid increases in levels of PUMA. Moreover, axonal branching of DRG neurons of Dusp $16^{-1-}$ mice was decreased, and the number of sensory nerves innervating the skin was around half compared with that of wild-type animals. Together, these findings suggest that DUSP16 acts as a negative regulator of the apoptotic system through PUMA and acts as a positive regulator of axon morphogenesis and sensory axon development.
The authors then turned their attention to PUMA. Trophic deprivation of cultured DRG explants from E13.5 $\mathrm{Puma}^{-/-}$mice resulted in microtubule depolymerization that was substantially lower than in controls. Moreover, $24 \mathrm{~h}$ after trophic deprivation, axons from wild-type mice had degenerated, but axons from the mutant mice were mostly protected and contained less active caspase 3 , suggesting that PUMA mediates axonal degeneration via caspase 3 .

To investigate the interaction between neuroprotective DUSP16 and pro-degenerative PUMA the authors generated double knockout mice. In these animals, caspase 3 activation and axonal degeneration following trophic withdrawal were delayed compared with controls, and the loss of axonal innervation observed in Dusp $16^{-/-}$mice was reversed in the double mutants. This suggests that PUMA acts downstream of DUSP16. Overall, these data suggest that developmental axon pruning is regulated by the balance of activity of proteins with opposing effects on axonal degeneration.

Sian Lewis

ORIGINAL ARTICLE Maor-Nof, M. et al. Axonal degeneration is regulated by a transcriptional program that coordinates expression of pro- and anti-degenerative factors. Neuron http://dx.doi. org/10.1016/j.neuron.2016.10.061 (2016) 